International Journal of Modern Physics A

(C) World Scientific Publishing Company

\title{
HYBRID AND CONVENTIONAL BARYONS IN THE FLUX-TUBE AND QUARK MODELS
}

\author{
PHILIP R. PAGE \\ Theoretical Division, MS B283, Los Alamos National Laboratory, Los Alamos, NM 87545, USA. \\ E-Mail: prp@lanl.gov
}

\begin{abstract}
The status of conventional baryon flux-tubes and hybrid baryons is reviewed. Recent surprises are that a model prediction indicates that hybrid baryons are very weakly produced in glue-rich $\Psi$ decays, and an analysis of electro-production data concludes that the Roper resonance is not a hybrid baryon. The baryon decay flux-tube overlap has been calculated in the flux-tube model, and is discussed here. The behavior of the overlap follows naïve expectations.
\end{abstract}

Keywords: baryon; flux-tube; hybrid; decay

\section{Update on previous reviews}

Comprehensive reviews on baryon flux-tubes and hybrid baryons are available. 112 Here developments since the last review in 2002 are summarized.

Baryon flux-tube: The consensus in quenched lattice QCD is that the baryon potential at sufficient distances is best described by the sum of a Coulomb, Y-shaped confinement, and constant term. $\frac{3}{3}$ This statement is independent of the lattice operator, and hence physical. 4 The difference with Abelian projected full lattice QCD is small. ${ }^{5}$ The baryon flux action density $[5$ is displayed in Fig. 10 The density clearly peaks at the three quarks and the junction. However, the detailed contour lines, as well as the Y-shape, are lattice operator dependent. 4 Abelian projected full lattice QCD shows $\$$ that cromo-electic flux flows in the same directions as the flux-tubes, with solenoidal currents circulating around them. This supports 5 the idea that QCD is a dual superconductor.

Hybrid baryon: The first quenched lattice QCD calculation of the low-lying hybrid baryon potential has been performed. $[6$ The lowest gluonic excitation energy (Fig. 2) is consistently greater than $1 \mathrm{GeV}$. This is several hundred MeV larger than the flux-tube model prediction 7 , which already gives the highest mass prediction for the lightest $N \frac{1}{2}^{+}$hybrid baryon of all the models 12 . If the lattice calculation is correct, this hybrid baryon will likely have a mass above $2 \mathrm{GeV}$, which would mean that the hybrid baryon spectrum starts at a very high mass. In contrast to this, QCD sum rule predictions for hybrid baryon masses remain low: Most recently, the $\Lambda \frac{1}{2}^{+}$is predicted $\frac{8}{6}$ at $1.6(2) \mathrm{GeV}$. A possible reason why the lattice calculation is high is that the effect of higher excited states has not been eliminated, although it 

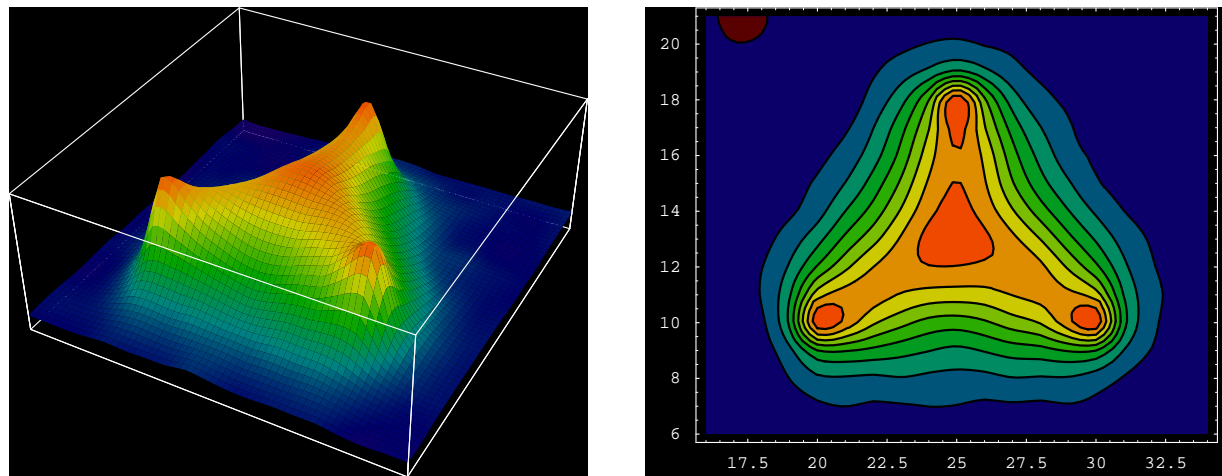

Fig. 1. Side view and top-down view of the flux action density.

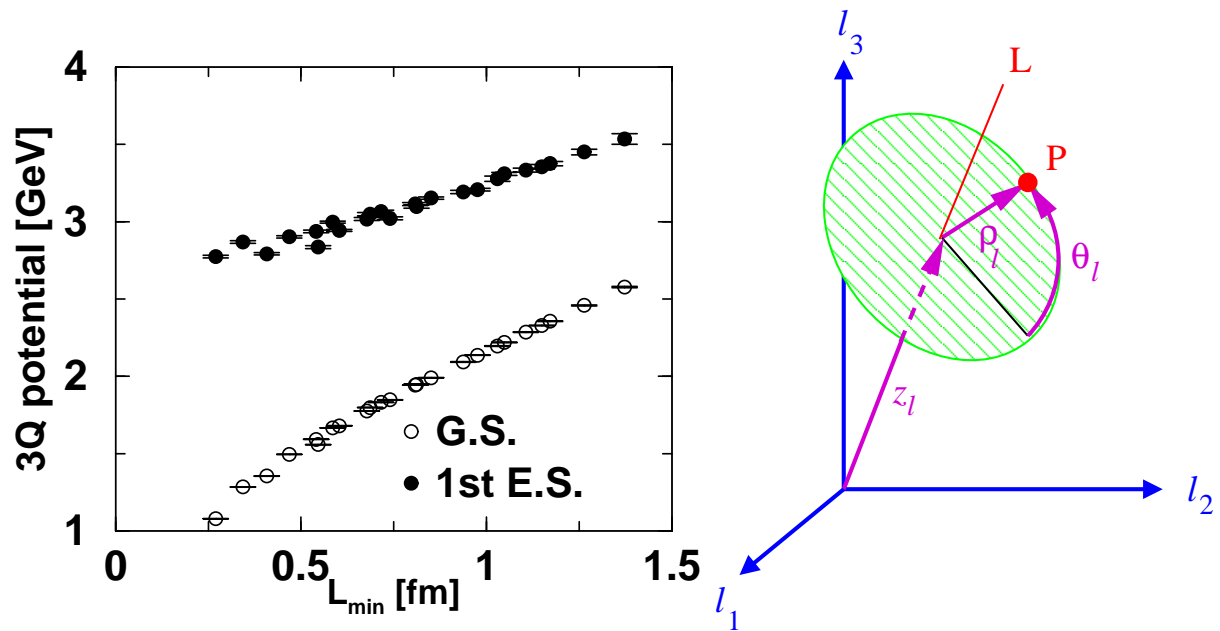

Fig. 2. Left is the baryon and lowest hybrid baryon potential as a function of $\mathrm{L}_{\min } \equiv l_{1}+l_{2}+l_{3}$. Here $l_{i}$ is the distance between quark $i$ and the junction equilibrium position, shown in Fig. 3 Right is a cylindrical coordinate system where the point $\mathrm{P}=\left(l_{1}, l_{2}, l_{3}\right)$ is rewritten in terms of $\rho_{l}, \theta_{l}$ and $z_{l}$. The line $l_{1}=l_{2}=l_{3}$ is denoted by $\mathrm{L}$.

is stated $[6]$ that this is not the case.

Hybrid baryon production in $\Psi$ decay, a glue-rich process, has long been thought to be promising. ${ }^{2}$ Particularly, BES at BEPC can look for $\Psi \rightarrow$ hybrid $\bar{N}$. Recently, several high mass peaks have been reported 9 : a $J^{P}=\frac{1}{2}^{+} 2 \sigma$ peak at $1834_{-55}^{+46} \mathrm{MeV}$ seen in $\Psi \rightarrow p \bar{p} \eta$, and a $2068 \pm 3_{-40}^{+15} \mathrm{MeV}$ peak seen in $\Psi \rightarrow N \bar{N} \pi$. A recent model calculation 10 finds, in contrast to naïve expectations, that hybrids are very weakly produced relative to radially excited baryons in $\Psi$ decay. This is due to a low probability of the gluon coming from the $\Psi$ to fold in to the qqqg hybrid wavefunction, and color factors in the hybrid baryon wave function. In fact, it is suggested that $\Psi$ decay is a filter in favor of conventional baryons. 10 


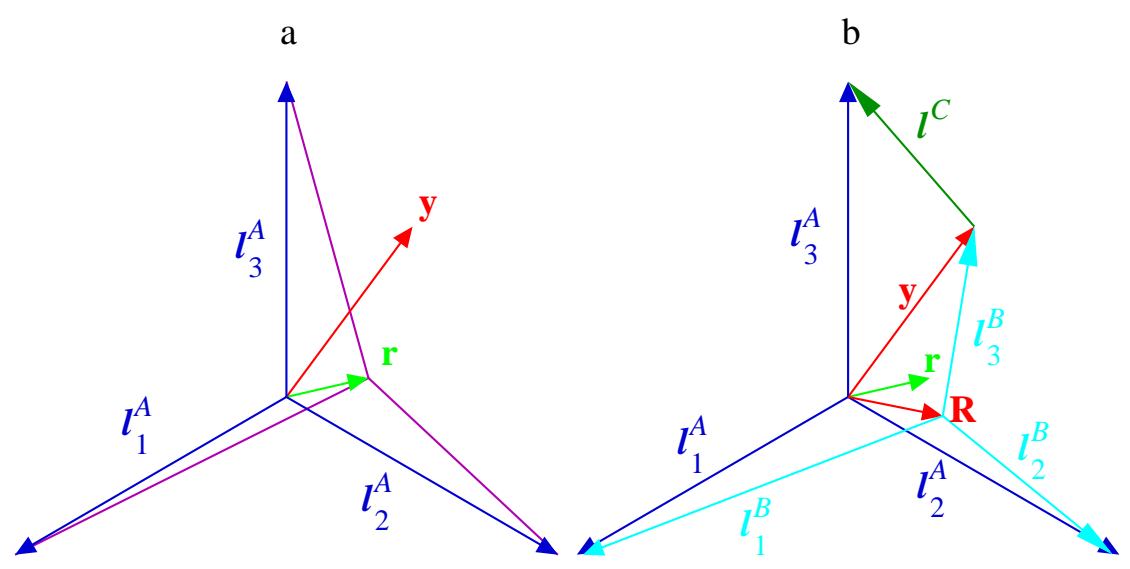

Fig. 3. Coordinate system described in the text. The length $l_{i}$ used in the text equals $\left|\boldsymbol{l}_{i}^{A}\right|$. The junction equilibrium position of the final baryon $B$ is denoted by $\mathbf{R}$.

Hybrid baryon electro-production: A recent analysis of new Jefferson Lab data finds 11 that the amplitude for Roper resonance $\left(N \frac{1}{2}^{+}(1440)\right)$ production via longitudinal photons is substantial, inconsistent with a model prediction that it should be zero. The Roper hence does not fit well as a hybrid baryon.

\section{Baryon decay flux-tube overlap}

When a baryon decays via OZI allowed quark-antiquark pair creation to a baryon and a meson, it has not hitherto been considered what happens to the flux-tubes. There is certainly an effect, as the pair is created from gluons. Here we report on the first preliminary calculation. Such a calculation exists for meson decay to two mesons. 1213 The structure of the decay can be investigated by fixing the initial quarks in the baryon, and evaluating the overlap of the initial flux configuration with the final baryon and mesons, for a fixed quark-antiquark pair created from the initial flux-tube: the "flux-tube overlap". This quantity enters in models of baryon decay where it is traditionally assumed to be a constant. This is equivalent to taking the Y-shaped flux structure to have no effect on decay. In such a picture the main effect of confinement on the decay is that it specifies the decay operator for quark-antiquark pair creation, e.g. the ${ }^{3} P_{0}$ decay operator 13 . This operator is typically taken to act locally with no knowledge of the global Y-shaped structure. Here the concern is not with the local character of decay (the decay operator), but with imposing constraints from Y-shaped confinement (the flux-tube overlap). The baryon decay flux overlap

$$
\begin{aligned}
\gamma\left(l_{i}, \mathbf{y}\right) \equiv \sum_{N^{C}=1}^{N_{3}^{A}-2} \int & d^{3} r\left(\prod_{i=1}^{3} \prod_{j=1}^{N_{i}^{A}} d^{2} y_{j}^{i A}\right) \delta^{2}\left(\mathbf{y}_{N^{C}+1}^{3 A}-\left(\mathbf{y}-\boldsymbol{l}_{3}^{A}-\frac{N^{C}+1}{N_{3}^{A}+1}\left(\mathbf{r}-\boldsymbol{l}_{3}^{A}\right)\right)\right) \\
& \times \psi^{A}\left(\mathbf{r}, \mathbf{y}_{j}^{i A}\right) \psi^{B *}\left(\mathbf{r}^{B}, \mathbf{y}_{j}^{i B}\right) \psi^{C *}\left(\mathbf{y}_{j}^{C}\right)
\end{aligned}
$$



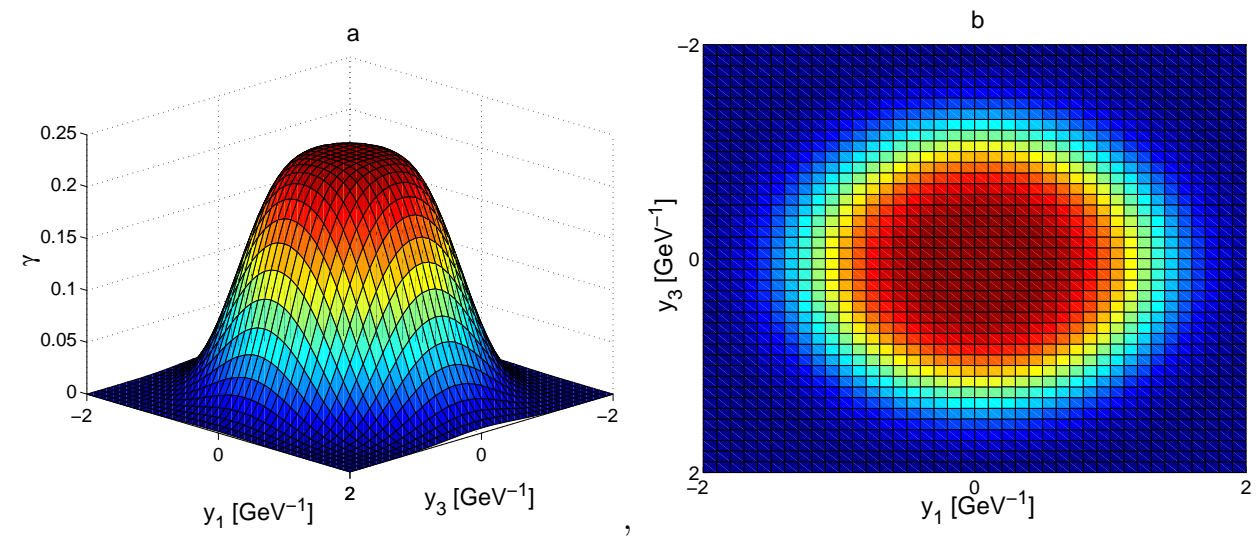

Fig. 4. (a) Side view and (b) top-down view of the baryon decay flux-tube overlap $\gamma$ in GeV. It is invariant under $y_{3} \rightarrow-y_{3}$ (there is nothing special about whether pair creation is above or below the plane of the three quarks), but the invariance under $y_{1} \rightarrow-y_{1}$ only ensues because $l_{1}=l_{2}$.

measures the overlap of the flux-tube wavefunction of initial baryon A, with those of final baryon $\mathrm{B}$ and meson $\mathrm{C}$, assuming that pair creation takes place at position y. The overlap is evaluated analytically in the flux-tube model of Isgur and Paton $\frac{714}{}$ as was done for the meson case 1213 . In this model the flux is represented by oscillating beads, and for the baryon the flux-tubes meet at a junction. The coordinates of the quarks and junction are displayed in Fig. 3(b). The position of the junction of the initial baryon, measured from its equilibrium position, is denoted by r. In Eq. 1 the position of bead $j$ associated with quark $i$ is denoted by $\mathbf{y}_{j}^{i}$. For the calculation shown, the constituent quark masses we taken to be $330 \mathrm{MeV}$, the string tension that of mesons $\left(0.18 \mathrm{GeV}^{2} \approx 1 \mathrm{GeV} / \mathrm{fm}\right), 714$ and all the bead masses equal. Bead masses are fixed by the condition that their sum equals the potential energy in the flux-tubes when the system is in equilibrium. The flux-tube overlap $\gamma$ depends on the six variables $\mathbf{y}$ and $l_{i}$, shown in Fig. 3(a). There is also dependence on the number of beads associated with each flux-tube in the initial baryon $\left(N_{i}^{A}\right)$. If pair creation can take place on more than one bead, $\gamma$ is the sum of the possible overlaps. (Hence the sum over the number of beads on the final meson $N^{C}$ in Eq. 1) Without loss of generality pair creation associated with the flux-tube of the third quark is studied. The components of $\mathbf{y}$ perpendicular to the vector $\boldsymbol{l}_{3}^{A}$ are $y_{1}$ (in the three-quark plane) and $y_{3}$ (perpendicular to the three-quark plane). The component of $\mathbf{y}$ along $\boldsymbol{l}_{3}^{A}$ is $y_{2}$. When any variables are fixed in the Figures, the following choices are made, unless indicated otherwise: $y_{1}=y_{3}=0, y_{2}=1.25$ $\mathrm{GeV}^{-1}=0.25 \mathrm{fm}, l_{i}=2.5 \mathrm{GeV}^{-1}=0.5 \mathrm{fm}$ and $N_{i}^{A}=3$.

The dependence of $\gamma$ on the pair creation position $\mathbf{y}$ is as follows. The dependence

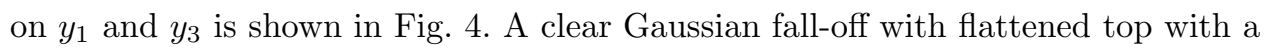
diameter $\sim 0.4 \mathrm{fm}$ think can be seen. This follows closely what would be expected for a thick flux-tube which falls off away from $\boldsymbol{l}_{3}^{A}$ as predicted by the flux-tube model (see Fig. 2 of Ref. 12, ). The Gaussian fall-off with flattened top continues to 
a
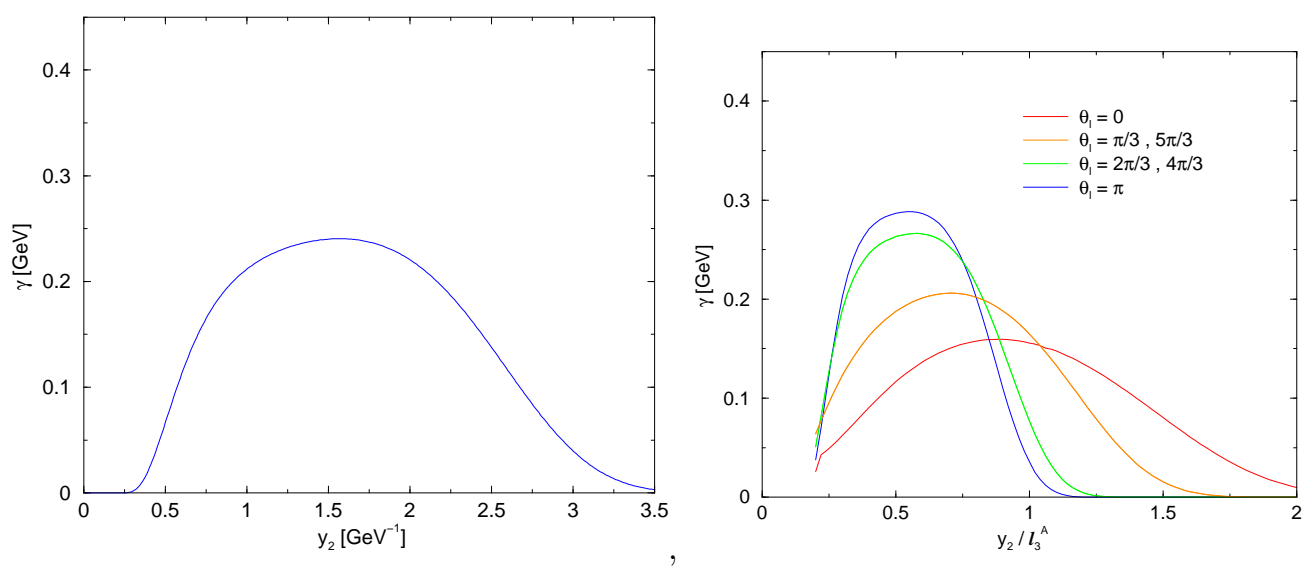

Fig. 5. The variation of $\gamma$ with $l_{i}$ shown in (b) is achieved by redefining $l_{i}$ in terms of $\rho_{l}, \theta_{l}$ and $z_{l}$ defined in Fig. 2 and plotting the variation as a function of $\theta_{l}$. The calculations are for $\rho_{l}=\rho \max / 2\left(\rho \max =z_{l} / \sqrt{2}\right)$ and $z_{l}=\sqrt{3} \times 2.5 \mathrm{GeV}^{-1}$.

be observed as $y_{2}$ is varied. Fig. 5 (a) shows the dependence on $y_{2}$. The asymmetric shape is because pair creation can take place beyond the position of the third quark $\left(y_{2}=l_{3}=2.5 \mathrm{GeV}^{-1}\right)$. Vanishing pair creation at $\mathbf{y}=\mathbf{0}$ is an artifact of the small-oscillations approximation $7 / 1213 / 14$ made in this calculation, and is not a prediction of the flux-tube model. The dependence of $\gamma$ on quark position $l_{i}$ variations is shown in Figs. [5) and 6 The extremes of the variation are consistently (a) smallest $\gamma$ when $l_{3}<l_{1}=l_{2}$ (red line $\theta_{l}=0$ ), and (b) largest $\gamma$ when $l_{3}>l_{1}=l_{2}$ (blue line $\theta_{l}=\pi$ ). These extremes are easily understood intuitively: When $l_{3}$ is small / large, there is less / more opportunity for pair creation associated with the third quark. Fig. 国 (b) shows that pair creation becomes more confined to the region inside the third quark $\left(0 \leq y_{2} \leq l_{3}\right)$ as $l_{3}$ increases. There is also a dependence on the number of beads $N_{i}^{A}$. Calculations were also performed for $N_{i}^{A}=4$. The number of beads determine the inter-bead spacing, a regularization parameter, which is unphysical. The qualitative features of the dependence of $\gamma$ on $\mathbf{y}$ and $l_{i}$ discussed above are independent of $N_{i}^{A}$, and hence physical. They are also without exception what one would have expected before the calculation was performed. The overall magnitude of decay is strongly dependent on $N_{i}^{A}$, and hence unphysical. This is as expected, since the magnitude of decay is normalized to phenomenology in pair creation models.

\section{Conclusions}

Baryon: At sufficient distances the potential is Y-shaped with a clear junction. The decay flux-tube overlap has been evaluated in the flux-tube model, and follows naïve expectations. Y-shaped baryon confinement can henceforth be incorporated 
a

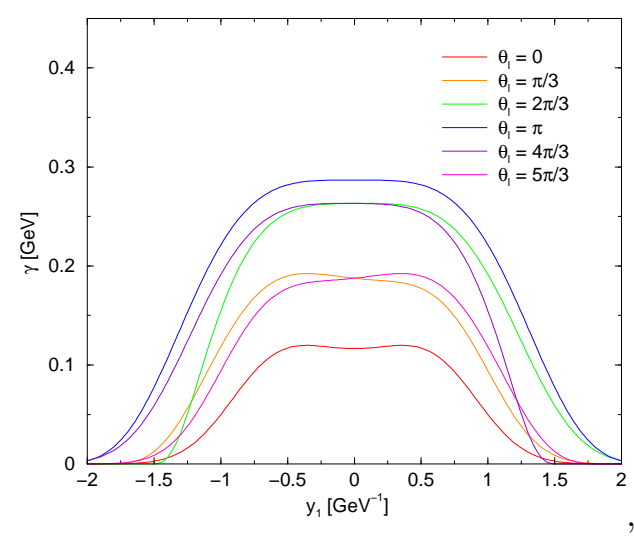

b

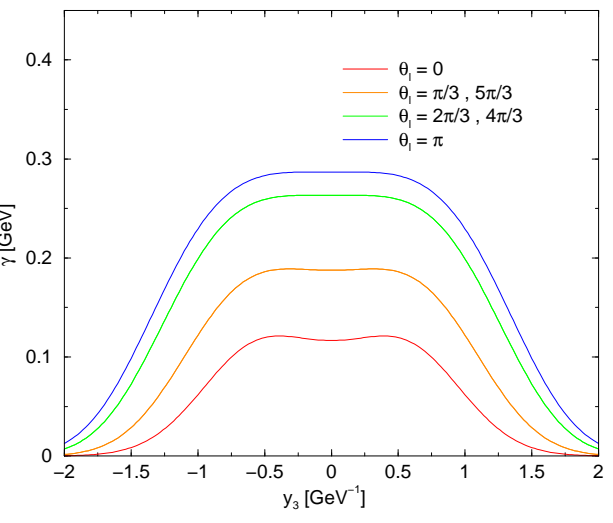

Fig. 6. Conventions as in Fig. 5 (b), except that $y_{2}=l_{3} / 2$.

in models of decay. Hybrid baryon: The only known way to study them rigorously is via excited adiabatic potentials. ${ }^{2}$ The low-lying state in models is $N \frac{1}{2}^{+}$with mass $1.5-1.8 \mathrm{GeV} .2$ If recent lattice QCD calculations are correct, this mass is most likely above $2 \mathrm{GeV}$. A recent model calculation shows that glue-rich $\Psi$ decay very weakly produces hybrid baryons. The Roper resonance does not appear to be a hybrid baryon from a recent analysis of electro-production via longitudinal photons.

\section{Acknowledgments}

Collaboration with N.F. Black (University of Tennessee, Knoxville) on the baryon decay flux-tube overlap, and hospitality at MENU2004, are acknowledged.

\section{References}

1. T. Barnes, contribution at the COSY Workshop on Baryon Excitations (May 2000, Jülich, Germany), nucl-th/0009011

2. P.R. Page, Proc. of the $9^{\text {th }}$ International Conference on the Structure of Baryons (BARYONS 2002) (March 2002, Newport News, VA), nucl-th/0204031.

3. T.T. Takahashi, H. Matsufuru, Y. Nemoto and H. Suganuma, Phys. Rev. Lett. 86, 18 (2001); Phys. Rev. 65, 114509 (2002); C. Alexandrou, P. de Forcrand and O. Jahn, Nucl. Phys. (Proc. Suppl.) 119, 667 (2003).

4. F. Okiharu and R.M. Woloshyn, Nucl. Phys. (Proc. Suppl.) 129, 745 (2004).

5. V.G. Bornyakov et al. (DIK Collab.), hep-lat/0401026

6. T.T. Takahashi and H. Suganuma, Phys. Rev. Lett. 90, 182001 (2003).

7. S. Capstick and P.R. Page, Phys. Rev. C66, 065204 (2002).

8. L.S. Kisslinger, Phys. Rev. D69, 054015 (2004).

9. H. Li et al. (BES Collab.), Phys. Lett. B510, 75 (2001); M. Ablikim et al. (BES Collab.), hep-ex/0405030

10. R.G. Ping, H.C. Chiang and B.S. Zou, nucl-th/0408007

11. I.G. Aznauryan et al., nucl-th/0407021 V.D. Burkert, these proceedings.

12. N. Dowrick, J. Paton and S.J. Perantonis, J. Phys., 423 G13 (1987).

13. R. Kokoski and N. Isgur, Phys. Rev. D35, 907 (1987). 
January 5, $2019 \quad$ 17:5 WSPC/INSTRUCTION FILE PRPage

Hybrid and Conventional Baryons 7

14. N. Isgur and J. Paton, Phys. Rev. D31, 2910 (1985). 


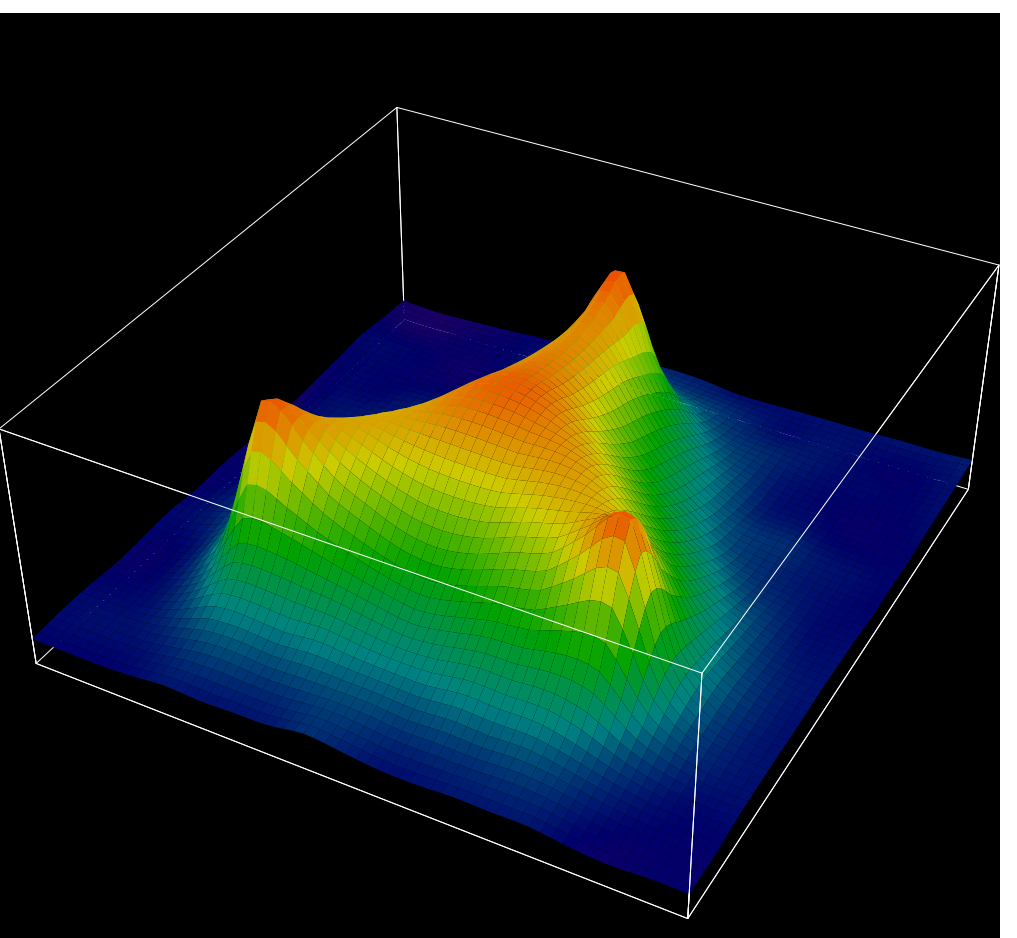

